\title{
CMOS Transmitter using Pulse-Width Modulation Pre-Emphasis achieving 33dB Loss Compensation at 5-Gb/s
}

\author{
J.H.R. Schrader, E.A.M. Klumperink, J.L. Visschers ${ }^{1}$, B. Nauta \\ IC Design Group, MESA+ Research Institute, University of Twente, Enschede, The Netherlands \\ ${ }^{1}$ NIKHEF, Amsterdam, The Netherlands \\ E-mail: j.h.r.schrader@utwente.nl
}

\begin{abstract}
A digital transmitter pre-emphasis technique is presented that is based on pulse-width modulation, instead of finite impulse response (FIR) filtering. The technique fits well to future high-speed low-voltage CMOS processes. A $0.13 \mu \mathrm{m}$ CMOS transmitter achieves more than $5 \mathrm{~Gb} / \mathrm{s}(2-\mathrm{PAM})$ over $25 \mathrm{~m}$ of standard RG-58U low-end coaxial copper cable. The test chip compensates for up to $33 \mathrm{~dB}$ of channel loss at the fundamental signaling frequency $(2.5 \mathrm{GHz})$, which is the highest figure compared to literature.

Keywords: pulse-width, modulation, pre-emphasis, transmitter, equalization, copper, cable and CMOS.
\end{abstract}

\section{Introduction}

High-speed data-communication over lossy copper channels suffers from Inter-Symbol Interference (ISI). In fig. $1 \mathrm{a}$, the magnitude transfer function $\left|\mathrm{S}_{21}\right|$ of $25 \mathrm{~m}$ RG-58U low-cost, low-end, standard coaxial cable is shown. It can be seen that the channel exhibits $31 \mathrm{~dB}$ of loss at the fundamental frequency $(2.5 \mathrm{GHz})$ for a $5 \mathrm{~Gb} / \mathrm{s} 2$-PAM signal. The pulse response of this cable to a 200ps polar Non-Return to Zero (NRZ) pulse (fig. 1b) shows a very long tail that will interfere with neighboring symbols. To compensate, transmitter pre-emphasis and/or receiver equalization is necessary $[1,2,3,4]$. The latter, receiver equalization, typically involves several analog blocks with speed, accuracy and linearity requirements. On the other hand, transmitter pre-emphasis allows the use of a simple receiver that only needs to sample binary values [4]. Pre-emphasis methods found in literature are commonly based on symbol-spaced finite impulse response (FIR) filtering $[1,2,3,4]$. In order to flatten the channel response, the transmitted low-frequency amplitude is attenuated to match the loss figure at the fundamental frequency.
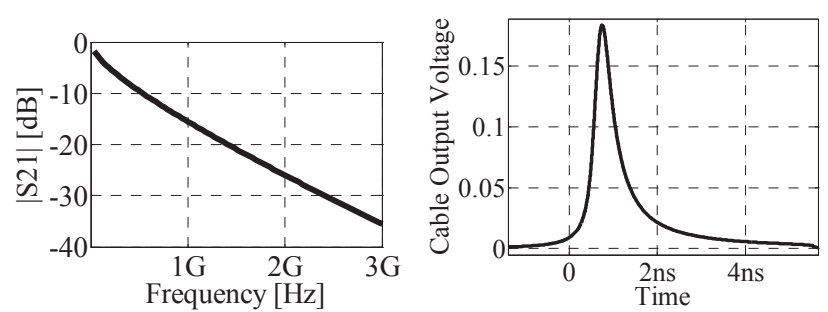

Fig. 1a (left): $\left|\mathrm{S}_{21}\right|$ of $25 \mathrm{~m}$ RG-58U low-cost, low-end standard coaxial cable. Fig $1 \mathrm{~b}$ (right): Cable response to a 200ps pulse.
The performance for 2-PAM pre-emphasis schemes can be evaluated by comparing the loss figure (in $\mathrm{dB}$ ) at the fundamental frequency (e.g. $2.5 \mathrm{GHz}$ for a $5 \mathrm{~Gb} / \mathrm{s}$ signaling rate), at which error free transmission is still possible. Different work can be compared on a loss figure basis, as long as the shape of the $S_{21}$ is comparable. This is approximately the case for all copper cables (either coaxial or twisted pair), assuming that the ratio between skin effect and dielectric loss is not extremely different. Such a comparison is better than a comparison on a cable length basis because the pre-emphasis designer faces the same challenge for a long low-loss cable as for a short high-loss (low-end) cable. In recent work, a combination of pre-emphasis and post-equalization has led to $27 \mathrm{~dB}$ $(18 \mathrm{~dB}+9 \mathrm{~dB})$ loss compensation at a signaling rate of $5 \mathrm{~Gb} / \mathrm{s}$ [1]. None of the pre-emphasis FIR filters that use 2 taps $[1,3,4]$ achieve more than $18 \mathrm{~dB}$. Using a more complex 5 -tap symbol-spaced FIR filter, a loss compensation of $30 \mathrm{~dB}$ has been achieved at $3.125 \mathrm{~Gb} / \mathrm{s}$ [2]. In this work, as a proof-of-principle, it will be shown that a very simple pulse-width modulation (PWM) scheme is a good alternative for providing pre-emphasis. A similar technique was recently proposed by our group for on-chip communication over $1 \mathrm{~cm}$ long RC-limited interconnects [5]. Our paper demonstrates the suitability of the PWM technique for transmission over $\sim 25 \mathrm{~m}$ low-end copper cables. This PWM pre-emphasis method for copper cables can achieve a loss compensation of $33 \mathrm{~dB}$ at $5 \mathrm{~Gb} / \mathrm{s}$, which is the highest figure compared to those found in literature.

\section{Pulse-Width Modulation Pre-Emphasis}

A 2-PAM transmitter without pre-emphasis would send only a plain polar NRZ pulse for every bit. However, the cable smears out the pulses (as shown in fig. 1b), leading to ISI. To compensate, a 2-tap symbol-spaced FIR filter sends a delayed (lower amplitude) inverse polarity pulse after each main NRZ pulse (during the next bit time). Loosely speaking, this inverse polarity pulse compensates for the energy in the long tail of the cable response, leading to a much narrower cable output pulse than for the transmitter without pre-emphasis. These 2-tap symbol-spaced FIR filters are easy to implement and generally used for pre-emphasis $[1,3,4]$. We observed that similar functionality might be obtained using a fixed amplitude, but variable (time-)width inverse pulse. In fig. 2, this is illustrated. In fig. 2a, the PWM transmitter pulse shapes are illustrated and in fig. $2 \mathrm{~b}$ the simulated cable responses (25m RG-58U) are shown. 

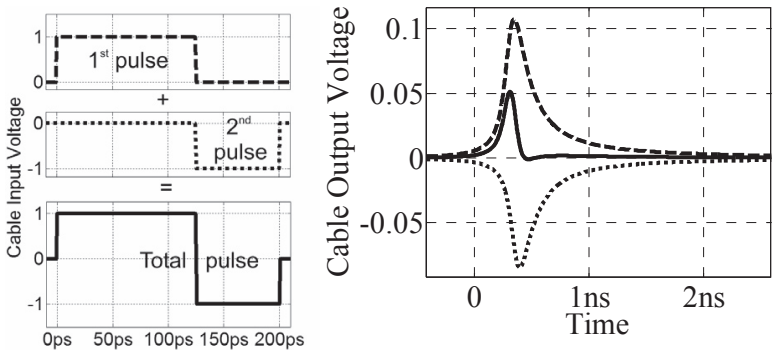

Fig 2a (left): Total PWM input pulse (solid) consists of $1^{\text {st }}$ and $2^{\text {nd }}$ pulse (dashed/dotted). Fig. 2b (right): Simulated cable responses.
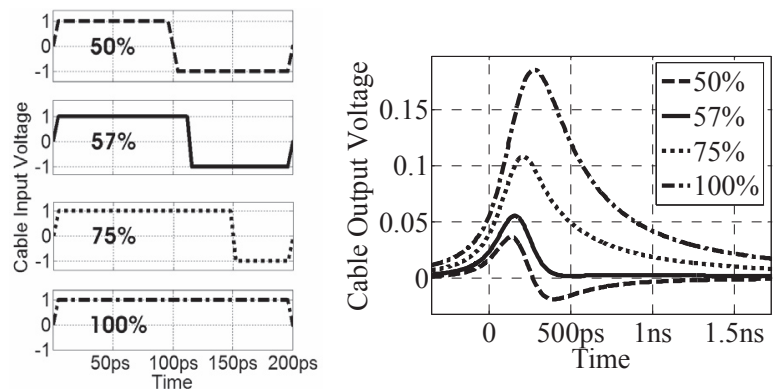

Fig 3a (left): PWM cable input pulse shapes for varying duty-cycles (200ps symbol duration). Fig 3b (right): Simulated cable responses (25m RG-58U) to PWM pulse shapes.

Simulations have been made with an accurate cable model that includes skin effect and dielectric loss [7,8]. For a clear understanding the (linear) cable responses to both the first pulse and the second inverse pulse have been calculated separately (fig. 2: dashed and dotted lines), and added up to see the combined effect (solid line). The transmit pulse can be shaped by adjusting its duty-cycle from $50 \%$ to $100 \%$, as is shown in fig. 3 (3a: cable input, $3 \mathrm{~b}$ : simulated output for $25 \mathrm{~m}$ RG-58U). A value of $100 \%$ corresponds to transmission of a normal (polar NRZ) data signal (no pre-emphasis, resulting in ISI), and $50 \%$ to transmission of a Manchester coded data signal. Note that, for the correct duty-cycle setting, the cable output pulse becomes much narrower than the response to a plain polar NRZ pulse. Second, note that, as can be seen from fig. 3, the optimum duty-cycle is in-between $50 \%$ and $100 \%$.

The PWM method does not tune the pulse amplitude (as for FIR pre-emphasis), but instead exploits timing resolution. This is beneficial in future high-speed low-voltage CMOS generations, and it allows (class-D) full switching to the supply voltages. Second, the implementation is very simple and can be low-area, low-power and digital. In comparison to 2-tap symbol-spaced FIR filters the PWM scheme has a higher switching frequency (due to the switching inside the symbol period) and spectral analysis shows that it achieves more high-frequency boost than 2-tap symbol-spaced filters, resulting in higher loss compensation.

\section{Circuit Building Blocks}

As shown in fig. 4 (operation principle), the pre-emphasis circuit XORs the data with a pulse-width modulated (PWM) clock in order to provide pre-emphasized data. The PWM clock is generated using an OR gate and a delay circuit.

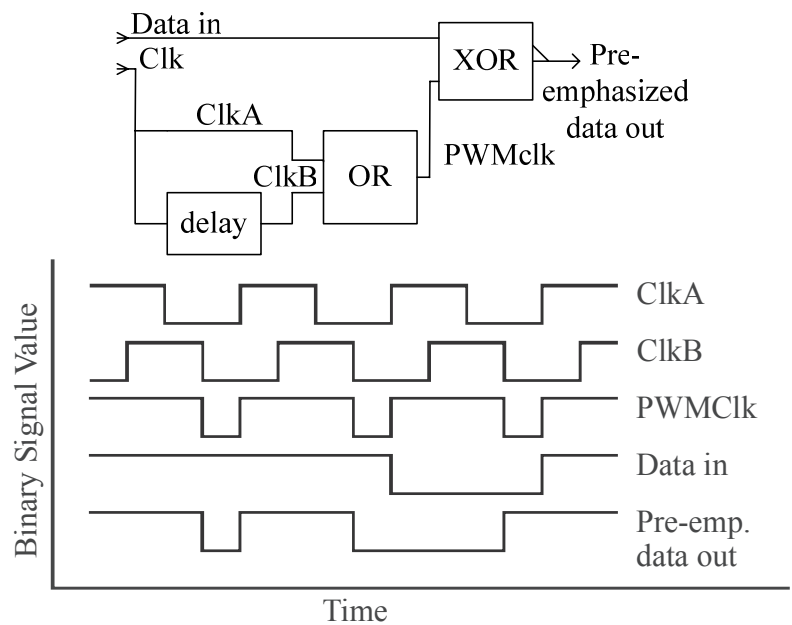

Fig. 4a (top): operation principle. Fig 4b (bottom): signals.

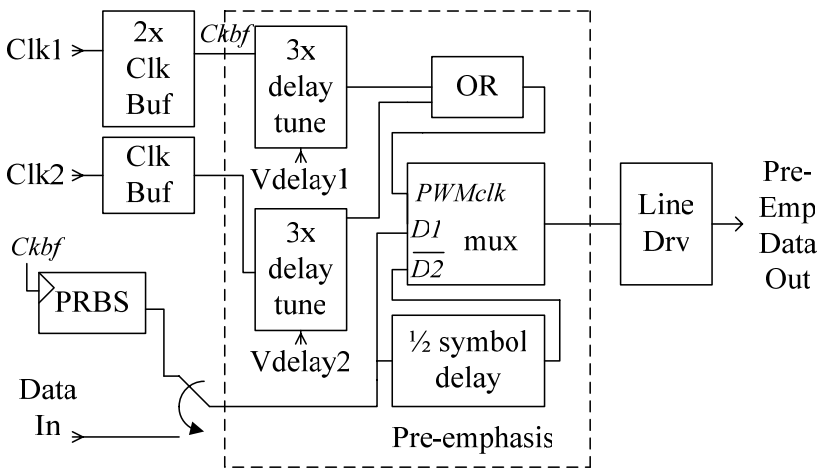

Fig. 5 Chip diagram: PRBS generator, clock buffers, pre-emphasis circuit and line driver. (All signals are differential).

In fig. 5 the chip diagram is shown. Because a short differential delay is easier to make than a single short delay, the relative delay for clock $\mathrm{B}$ is created by delaying clk 1 with delay1 and delaying clk2 with delay2. The differential delay is thus equal to (delay1-delay2). The XOR is implemented using a multiplexer (fig. 6a) that selects either D1 (non-inverted data) or D2 (inverted data). For optimum timing margin, $D 2$ is delayed half a symbol time using a negative edge clocked flip-flop. The duty-cycle of the PWM pulse shape can be tuned between $50 \%-100 \%$, when the relative phase-shift between clocks is $0^{\circ}-180^{\circ}$.

\section{A. Delay Circuit}

The time-shifted clock is generated using a variable delay circuit (fig. 6b) [6]. This circuit has a delay from in to out, which is mainly determined by the RC time at the output. By adding a negative resistance (positive feedback circuit in parallel to the output), the effective $\mathrm{R}$ can be changed and hence the RC-delay. The value of the negative resistance is controlled by the differential delay control voltage $\mathrm{V}_{\text {delP }}-\mathrm{V}_{\text {delN }}$, which divides the total bias current between the input differential pair and negative resistance pair. For $\mathrm{V}_{\text {delP }}$ " $\mathrm{V}_{\text {delN }}$, the delay is minimized. As the total bias current through the output resistors is fixed, the output swing remains constant. The required tuning range of the delay-circuit depends on the desired symbol length and on the necessary duty-cycle range for pre-emphasis. The (continuous) tuning range can be enlarged by cascading 
multiple delay stages. For very large delay ranges this becomes unpractical and it is more effective to combine (continuous tunable) delay with (discrete fixed) delay steps. In our prototype design, we aimed for flexibility to evaluate the new PWM-concept in various ways. Therefore we allow for providing external clocks, e.g. to accommodate for very low bit rates for long poor cables. During normal operation, both inputs $c l k 1$ and $c l k 2$ (fig. 5) can just be connected to the same clock.

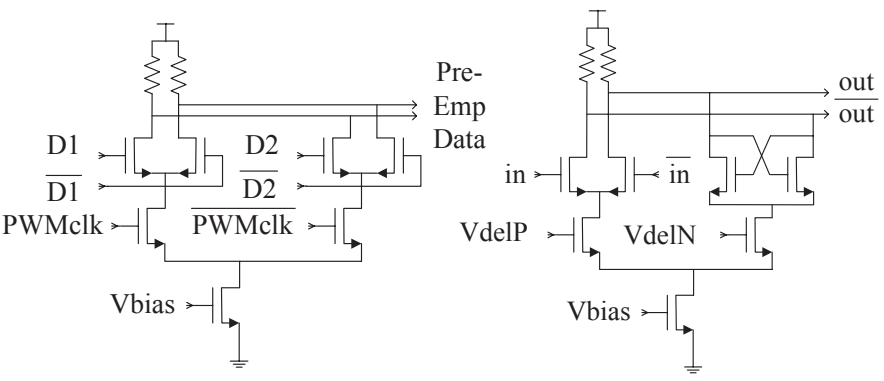

Fig. 6a (left): CML multiplexer. Fig. 6b (right): CML delay tuning.

The whole test chip has been designed in CML to provide maximum supply noise rejection and minimum supply noise injection and keep timing noise as low as possible. Also, this guarantees equal up- and down- slew rates.

\section{B. Line Driver}

The line driver (fig. 7) consists of three stages. Each stage has three times the $\mathrm{W} / \mathrm{L}$ dimensions and one third the resistance value of its predecessor. The final stage has $50 \Omega$ on-chip output resistance and a tail current of $24 \mathrm{~mA}$. Nominal single-ended output swing is $600 \mathrm{mV}_{\mathrm{p}-\mathrm{p}}$ (Differential 1.2 $\mathrm{V}_{\mathrm{p}-\mathrm{p}}$ )

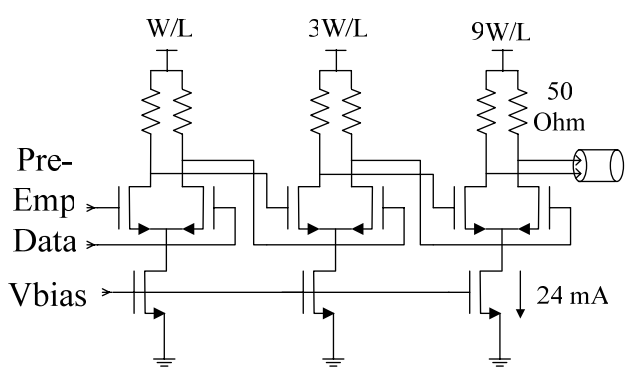

Fig. 7 Three-stage differential line driver.

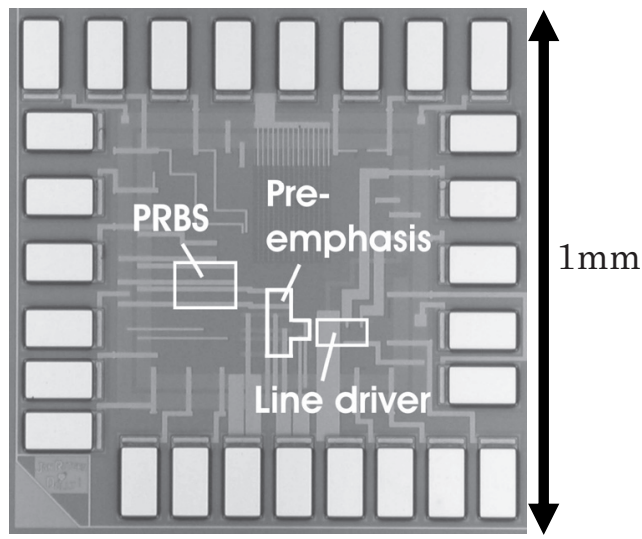

Fig. 8 Chip microphotograph.

\section{Measurement Results}

In fig. 8, a chip microphotograph is shown. Measurements have been made using an Agilent Digital Communication Analyzer (86100A) and an Anritsu pattern generator and BER tester (MP1632C with $2^{31}-1$ PRBS). Eye diagrams have been generated at all speeds. An on-chip pattern generator was provided $\left(2^{7}-1\right.$ PRBS) because the available external BER tester and pattern generator had a maximum speed of only $3.2 \mathrm{~Gb} / \mathrm{s}$.

Due to restrictions in measurement equipment and time limitations, no measurements could be made yet with twisted pair cable, however the transmitter has a differential output. So far only single-ended measurements have been made with coaxial RG-58U cable, using only one of the two transmitter outputs and terminating the other at $50 \Omega$. The cable was connected to the test chip using a $50 \Omega$ differential probe (with 4 pins: ground-signal-signal-ground). The RG-58U cable is very low-cost, low-end, and standard. All chip I/Os have on-chip $50 \Omega$ termination and are ESD protected.

\section{A. Effect of Adjustments in PWM Duty-Cycle}

In fig. 7, the transmitter output eyes are shown for different duty-cycles. The left- and right edges in the eye diagrams correspond to the symbol edges. (Compare to fig. 2a).

In fig. 8 the responses of a $10 \mathrm{~m}$ RG-58U cable to the pre-emphasized data stream with different pre-emphasis duty-cycles are shown. It can be seen that there is an optimum duty-cycle (middle figure). Under-emphasis is shown in the left figure and over-emphasis in the right figure. Note that the time scale in fig. 7 and fig. 8 is the same.

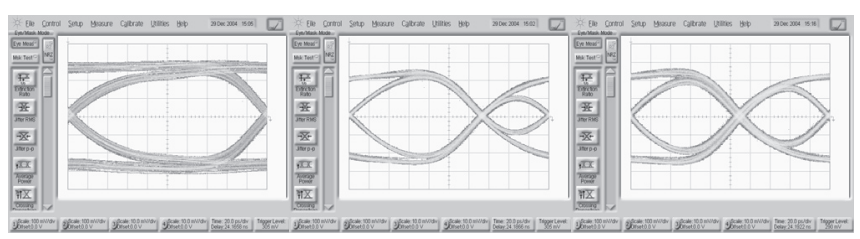

Fig. 7 Measured transmitter eyes at $5 \mathrm{~Gb} / \mathrm{s}$ with three different duty-cycle settings; left: no pre-emphasis (100\%), middle: weak pre-emphasis (66\%), right: strong pre-emphasis (55\%). Horizontal axis $=20 \mathrm{ps} / \mathrm{div}$, vertical axis $=100 \mathrm{mV} / \mathrm{div}$.

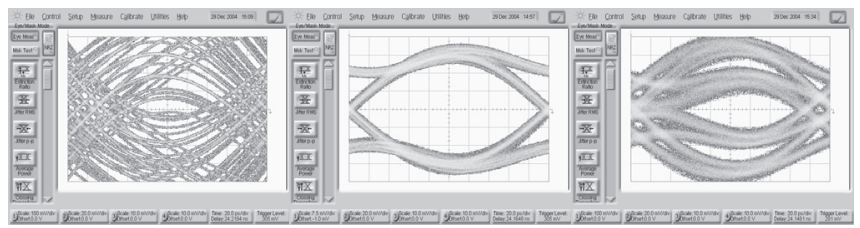

Fig. 8 Measured eyes of cable response for transmitter settings shown in previous figure and $5 \mathrm{~Gb} / \mathrm{s}$ over $10 \mathrm{~m}$ RG-58U cable. Horizontal axis $=20 \mathrm{ps} / \mathrm{div}$, vertical axis $=20 \mathrm{mV} / \mathrm{div}$.

\section{B. Eye Diagrams for $25 m R G-58 U$}

In fig. 9a $(4 \mathrm{~Gb} / \mathrm{s})$ and fig. $9 \mathrm{~b}(5 \mathrm{~Gb} / \mathrm{s})$, measured eye diagrams of the cable output for $25 \mathrm{~m}$ RG-58U are shown. These two speeds are shown to illustrate the difference in eye shape. The cable loss at $2.5 \mathrm{GHz}$ is $31 \mathrm{~dB}$, and the total channel loss is approximately $33 \mathrm{~dB}$ including additional parasitic losses in the path from chip to coaxial cable (probes, short wire, bias tee and connectors). Using a high-speed 
limiting amplifier and the BER tester, the BER has been tested up to $3.2 \mathrm{~Gb} / \mathrm{s}$ (due to limited speed of BER tester) and is $<10^{-12}$. From the clearly open eye diagrams it can be concluded that the pre-emphasis compensates enough channel loss to enable error free transmission at $5 \mathrm{~Gb} / \mathrm{s}$.

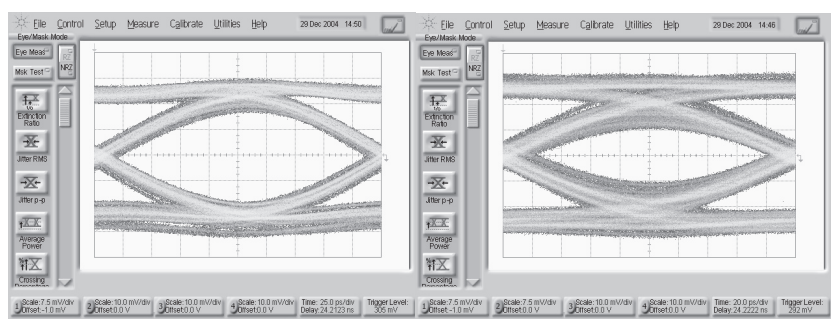

Fig. 9a (left): Measured output eye of $25 \mathrm{~m} \mathrm{RG-58U}$ at $4 \mathrm{~Gb} / \mathrm{s}$. Horizontal axis $=20 \mathrm{ps} /$ div, vertical axis $=10 \mathrm{mV} /$ div. Fig. $9 \mathrm{~b}$ (right): Measured output eye of $25 \mathrm{~m} \mathrm{RG}-58 \mathrm{U}$ at $5 \mathrm{~Gb} / \mathrm{s}$. Horizontal axis $=20 \mathrm{ps} / \mathrm{div}$, vertical axis $=7.5 \mathrm{mV} / \mathrm{div}$.

At a channel loss of $33 \mathrm{~dB}$, the small cable output amplitude puts a high demand on receiver sensitivity and it might be necessary to use differential signaling. Using the fully differential transmitter capabilities would boost the (differential) swing at the cable output with $6 \mathrm{~dB}$ while also rejecting common mode noise.

TABLE I

PRE-EMPHASIS COMPARISON WITH OTHER WORK

\begin{tabular}{|l|l|l|l|l|}
\hline Ref. & R & Loss & Ft. size & Type \\
\hline$[1] \mathrm{TX}$ only & $5 \mathrm{~Gb} / \mathrm{s}$ & $18 \mathrm{~dB}$ & $0.13 \mu \mathrm{m}$ & 2-tap FIR \\
\hline$[2] \mathrm{TX}$ only & $3.125 \mathrm{~Gb} / \mathrm{s}$ & $30 \mathrm{~dB}$ & $0.11 \mu \mathrm{m}$ & 5-tap FIR \\
\hline$[3]$ & $8 \mathrm{~Gb} / \mathrm{s}$ & $\sim 10 \mathrm{~dB}$ & $0.3 \mu \mathrm{m}$ & 2-tap FIR \\
\hline$[4]$ & $4 \mathrm{~Gb} / \mathrm{s}$ & $\sim 10 \mathrm{~dB}$ & $0.25 \mu \mathrm{m}$ & 2-tap FIR \\
\hline this work & $\mathbf{5 G b} / \mathbf{s}$ & $\mathbf{3 3 d B}$ & $\mathbf{0 . 1 3} \boldsymbol{\mu m}$ & PWM \\
\hline
\end{tabular}

TABLE II

ELECTRICAL CHARACTERISTICS OF TRANSMITTER

\begin{tabular}{|l|l|}
\hline Baudrate (2-PAM) & 5GBd \\
\hline U-I & $200 \mathrm{ps}$ \\
\hline TX amp. ( $\mathbf{V}_{\mathbf{p}-\mathbf{p}}$ ) nom. & $1.2 \mathrm{~V}$ (dif), \\
& $600 \mathrm{mV}$ (single-ended) \\
\hline Channel loss @ 2.5GHz & $33 \mathrm{~dB}$ \\
\hline $\mathbf{V}_{\text {sup }}$ & $1.2 \mathrm{~V}$ \\
\hline Power (pre-emphasis) & $12 \mathrm{~mW}$ \\
\hline Power (line driver) & $42 \mathrm{~mW}$ \\
\hline Power (clock buffering) & $39 \mathrm{~mW}$ \\
\hline Power (on-chip PRBS) & $17 \mathrm{~mW}$ \\
\hline
\end{tabular}

In table I, a comparison with other published work is given. It is shown that this work achieves the highest loss compensation $(33 \mathrm{~dB})$ at a bit rate of $5 \mathrm{~Gb} / \mathrm{s}$. From $[1,2]$ only the transmitter pre-emphasis has been taken into account (not the receiver equalizer). In table II, the electrical characteristics are given. Power is hard to compare because most publications only give total figures. In our current proof-of-principle design, the clock-buffering takes quite a lot of the power budget, which can be improved if internal clocks are available in the IC, like in practical applications. Because of the simplicity of the pre-emphasis method, area and power can be very small.

\section{Conclusions}

A new digital pre-emphasis technique based on pulse-width modulation (PWM) is introduced. The PWM method does not tune the pulse amplitude (as for FIR pre-emphasis), but instead exploits timing resolution. This fits well to future low-voltage high-speed CMOS processes. Using only single-ended measurements due to limitations in measurement equipment, successful transmission of a 2-PAM $5 \mathrm{~Gb} / \mathrm{s}$ data signal over $25 \mathrm{~m}$ of low-cost, low-end, standard RG-58U coaxial cable is demonstrated. This corresponds to a loss compensation of $33 \mathrm{~dB}$ at the fundamental frequency of $2.5 \mathrm{GHz}$, which is the highest figure compared literature. Main building blocks of the CML pre-emphasis circuit are a tunable delay, an OR gate and a multiplexer. The pre-emphasis technique is simple and can be implemented using only low power and area.

\section{Acknowledgements}

The authors would like to thank Stichting FOM (funding), CERN and Giovanni Cervelli (organizing chip fabrication), Daniel Schinkel, Paulo Moreira and Hans Verkooijen (helpful discussions), Henk de Vries, Gerard Wienk and Joop Rövekamp (practical assistance), and Lanzarote for the beautiful views.

\section{References}

[1] Y. Kudoh, M. Fukaishi and M. Mizuno, "A 0.13- $\mu \mathrm{m}$ CMOS 5-Gb/s 10-m 28AWG cable transceiver with no-feedback-loop continuous-time post-equalizer", IEEE J. Solid-State Circuits, vol. 38, pp. 741-746, May 2003.

[2] W. Gai, Y. Hidaka, Y. Koyanagi, J.H. Jiang, H. Osone and T. Horie, "A 4-channel $3.125 \mathrm{~Gb} / \mathrm{s} / \mathrm{ch}$ CMOS transceiver with $30 \mathrm{~dB}$ equalization", Symposium on VLSI Circuits, Digest of Technical Papers, pp. 138-141, 2004.

[3] R. Farjad-Rad, C.K. Yang, M. Horowitz, and T. Lee, "A $0.3-\mu \mathrm{m}$ CMOS $8 \mathrm{~Gb} / \mathrm{s}$ 4-PAM serial link transceiver", IEEE J. Solid-State Circuits, vol. 35, pp. 757-764, May 2000.

[4] M. Lee, W. Dally and P. Chiang, "Low-power area-efficient high-speed I/O circuit techniques", IEEE J. Solid-State Circuits, vol. 35, pp. 1591-1599, Nov. 2000.

[5] D. Schinkel, E. Mensink, E. Klumperink. E. van Tuijl, B. Nauta, "A $3 \mathrm{~Gb} / \mathrm{s} / \mathrm{ch}$ transceiver for RC-limited on-chip interconnects", IEEE International Solid State Circuits Conference 2005, in press.

[6] B. Razavi, "Design of analog CMOS integrated circuits", McGraw-Hill, 2001.

[7] F.E. Gardiol, "Lossy transmission lines", Artech House, 1987.

[8] P. Grivet and P. W. Hawkes, "The physics of transmission lines at high and very high frequencies", Academic Press, 1970. 